Jacques Guilhaumou, UMR « Triangle », Université de Lyon, CNRS/ENS-LSH

Vient de paraître dans Actuel Marx., N43, 2008, p. 29-42.

\title{
Le non-dit de l'idéologie : l'invention de la chose et du mot.
}

Au premier abord, la formulation néologique de la notion d'idéologie par Destutt de Tracy en 1797-1798 pour désigner la nouvelle « science des idées » au fondement de « la science de l'homme " semble intervenir dans un ciel serein, garanti par la relative stabilité de la République directoriale, où règne la seule passion du savoir. Cependant l'opposition très marquée, dès le départ de la formulation de l'idéologie, à la métaphysique politique du moi et de son activité telle qu'elle s'est déployée dans les années 1770-1780, puis imposée au début de la Révolution française, nous incite à aller au-delà du comment se positionne le terme d'idéologie dans la théorie des Idéologues ce qui s'avère assez vite dit, nous le verrons. Il est tout aussi important d'interroger le pourquoi d'une telle disgrâce «idéologique » de la métaphysique politique, et ceci pour des raisons bien particulières qui nous obligent à un détour par la conjoncture des années 1770-1780 où s'impose un travail novateur de l'esprit politique sur la base d'un « socle sociologique » constitutif de l'expérience révolutionnaire. Avec l'introduction du terme d'idéologie, c'est l'eau du bain (le socle sociologique) qui est jetée avec le bébé (la métaphysique politique) si l'on peut dire. Il ressort alors de ce cet usage néologique de la notion d'idéologie un effet apparent de rupture qui tient au contexte historique très particulier de son énonciation.

\section{I - Préalable à l'idéologie : le travail de l'esprit politique}

Au cours des années 1770-1780, moment proto-politique de la Révolution française au sens large ${ }^{1}$, la spectaculaire promotion de l'observation sociale, sous l'égide de divers événements ( de la "guerre des farines » à la convocation des Etats Généraux), de divers penseurs (Condillac, Mably, Helvétius, D’Holbach, Sieyès, etc.) et de diverses notions (peuple, société, opinion publique, ordre social), ouvre la possibilité d'une réflexion sur les modalités ontologiquement subjectives de la construction sociale au sein de l'esprit humain. Tout au long d'un processus historique qui nous mène de l'échec des réformateurs face à la résistance d'un «peuple raisonnable » (Mably) au cours de la «la guerre des farines » au climat pamphlétaire des années 1788-1789 à l'occasion de la convocation et de la réunion des Etats Généraux, la génération des penseurs de cette période des Lumières tardives d'une part affirme un principe d'activité individuelle à la base de la connaissance, d'autre part promeut l'usage de l'artifice pour permettre au travail de l'esprit de nous faire accéder à la libre disposition d'objets notionnels inédits au titre du nouvel ordre social, mais dans un continuum avec la réflexion sur le rapport d'une telle intelligence souveraine aux lois de la nature ${ }^{2}$. L'opinion publique prend alors un tour plutôt fictionnel en s'appuyant sur un appareil mental perçu de façon à la fois interne et concrète. Ainsi se met en place une métaphysique du moi en acte au fondement même de la nouvelle langue politique de 1789 .

L'opinion publique, une fiction concrète.

\footnotetext{
${ }^{1}$ Nous avons précisé l'importance de ce moment dans la première partie de notre récente synthèse sur « la langue politique de la Révolution française », Langage \& Société, $\mathrm{N}^{\circ} 113$, septembre 2005.

2 Sur ce dernier point, voir l'ouvrage d'André Charrak, Contingence et nécessité des lois de la nature au XVIIIème siècle. La philosophie seconde des Lumières, Paris, Vrin, 2006.
} 
A ce titre, l'opinion publique à la fin du $18^{\text {ème }}$ siècle ne procède plus du simple fait d'un développement autonome des espaces de conversation littéraire et de débat critique, souvent assimilés aux salons. Elle ne se confond pas non plus avec la civilité issue d'institutions publiques, telles que les académies, les sociétés philanthropiques et les loges maçonniques. A distance de ces conceptions soit idéales, soit concrètes de l'opinion publique, il s'agit d'ouvrir l'opinion publique à un espace de reconnaissance sociale ${ }^{3}$.

Certes, déjà la période très active des Encyclopédistes en matière éditoriale au cours des années 1750 et 1760 avait introduit un geste critique au sein de l'espace public mais en grande part par le fait de la conscience d'une solidarité entre philosophes des Lumières. Mais, dans les années 1770 et 1780 de consécration de la nouvelle sociabilité, l'opinion publique apparaît encore plus nettement chez les élites comme un espace de "sociétés particulières 》 - à l'exemple des salons ${ }^{4}$ - où se déploie une mondanité des hommes de lettres qui se structure dans des rapports hiérarchisés de reconnaissance. Cependant, au-delà d'une telle modalité de visibilité sociale, c'est la question de l'utilité sociale qui prend une part prépondérante dans l'observation sociale et contribue ainsi à la formation d' " un socle sociologique » - Sieyès invente le terme sociologie dans les années $1780^{5}$ - participant de l'invention de la société dans sa confrontation singulière avec l'individu particulier. D'ailleurs, la mise en place d'un tel socle sociologique, et de son corollaire une manière autre d'observer la société, permettent de façon décisive la multiplication des référents sociaux de l'opinion publique, de leur autoconstitution à l'exemple de l'abord singulier du peuple dans ses jugements particuliers, donc sorti du carcan de l'essentialisme, et au plus près d'une approche nominaliste de la société ${ }^{6}$.

En effet, le peuple d'abord défini négativement par les élites dans un espace de néantisation sociale, prend une certaine visibilité dans des appartenances sociales situées à distance des apparences et au plus près des mœurs populaires, désormais assignables à l'ordre social ${ }^{7}$. Le problème se pose alors de savoir comment s'articulent cette émergence d'une culture pragmatique du possible en société, donc située au plus des mœurs sociaux, tant dans le peuple que chez les élites, et l'invention concomitante, chez des auteurs savants (Condillac, D'Holbach, Helvétius, Sieyès etc.), d'une nouvelle culture critique de l'artifice politique qui introduit à l'ordre du pensable dans cette même période nominaliste des années 1770-1780.

S'il existe donc bien un usage public de la critique, il s'opère de plus en plus, au cours des années 1770 et 1780, dans le passage d'un monde de la société en construction vers un monde

${ }^{3}$ Nous avons déjà abordé la question de la reconnaissance sociale dans notre étude, « La Révolution française à l'horizon du mouvement social. Une question de visibilité sociale », Réseaux, $\mathrm{N}^{\circ} 129-130,2005$.

${ }^{4}$ Voir, à ce sujet, Antoine Lilti, Le monde des salons. Sociabilité et mondanité à Paris au XVIIIème siècle, Paris, Fayard, 2005

${ }^{5}$ Pour une approche philologique de la formation de ce néologisme, voir notre article. "Sieyès et le non-dit de la sociologie : du mot à la chose », Revue d'histoire des sciences humaines, $\mathrm{N}^{\circ} 15,2006$, et plus largement l'étude de Jean-Louis Morgenthalter sur "Condorcet, Sieyès, Saint-Simon et Comte. Retour sur une anamorphose », Socio-logos, revue électronique, $\mathrm{N}^{\circ} 2,2007$.

${ }^{6}$ Voir Laurence Kaufmann et Jacques Guilhaumou, L'invention de la société. Nominalisme politique et science sociale au XVIIlème siècle, Paris, EHESS, collection "Raisons pratiques », 2003.

Cf. Déborah Cohen, Le peuple : de l'Autre au différent. La construction des identités individuelles et collectives des classes populaires (France, XVIIIème siècle), Thèse de Doctorat de l'EHESS, sous la dir. d'Arlette Farge, 2004. 
de l'esprit en réalisation, donc de manière foncièrement intentionnelle. D'ailleurs l'époque invente un nouveau mot, la socialité, pour désigner la relation de l'individu à la totalité sociale, au-delà du fait naturel de la mutualité sociale propre à la sociabilité. Si l'homme agit et réfléchit sur «une base sociologique», il l'est donc non seulement par le fait de la sociabilité, qui désigne sa capacité (sociale) à répondre des fins d'utilité (sociale), mais aussi par une capacité intellectuelle de concevoir et de réaliser en esprit l'ordre social, d'en exprimer le tout, donc de faire oeuvre de socialité.

A ce titre, le débat critique se traduit dans une fiction concrète qui se décline plus au singulier qu'il ne renvoie à une réalité pratique au sein d'un espace de sociabilité, ce qu'il a majoritairement été du temps des Encyclopédistes lorsque ces philosophes ont initié leur contemporain aux diverses activités «libres» de l'homme sous l'impulsion de nouvelles disciplines $^{8}$. L'opinion publique prend donc de plus en plus un tour artificialiste par sa capacité à rendre auto-intelligible les rapports sociaux dans des notions-concepts qui nomment le nouvel ordre social ${ }^{9}$. La mondanité concrète de la conversation intellectuelle ne peut ainsi se confondre avec l'échange des discours savants, de nature particulièrement artificialiste le temps de l'événement en pensée - de l'événement cognitif - qui produit de nouvelles nominations sociales et politiques. La puissance d'évocation de l'opinion publique rencontre ainsi un appareil mental dont nous allons préciser les caractéristiques.

L'internalité de l'appareil mental : l'activité du moi.

Notre réflexion présente se centre plus largement sur le problème de la connexion empirique entre la réalité et le discours ${ }^{10}$, au titre à la fois de l'insistance sur la matérialité des sensations originaires au sein de l'esprit et l'importance de la liaison des signes dans la langue. $L$ 'appareil mental est donc perçu avant tout dans son fonctionnement interne. Du monde vers l'esprit, la conscience du moi internalise la réalité des choses, la reconfigure, par la multiplication des relations et des événements en esprit via la puissance de la liaison des signes et le pouvoir de l'imagination. La classification cognitive procède alors d'un lien nécessaire entre l'ordre rationnel et l'ordre de l'expérience, avec une part importante accordée à la faculté de langage

Nous sommes ici dans l'espace de traduction du paradigme lockien de la connexion mentale (toute catégorisation mentale inclut des mots, des relations et des événements) dans une procédure sensualiste de la formation des connaissances, avec Condillac principalement. A ce titre, la métaphysique est réduite à la reprise réflexive par l'esprit des faits donnés par l'expérience ${ }^{11}$. Il est alors possible de développer une sémantique cognitive qui s'interroge sur la pertinence ou non d'une métaphysique du moi et de son activité. Nous pouvons ainsi retracer la généalogie d'une métaphysique du moi des Encyclopédistes à Maine de Biran, dont les Idéologues seront les principaux adversaires

\footnotetext{
${ }^{8}$ D'après Martine Groult, « Dans la création des disciplines, il y a l'affirmation selon laquelle la classification introduit une méthode qui place les matières selon un ordre dont l'homme produit la démonstration du sens », in La création des disciplines, sous la dir. de M. Groult, Paris, CNRSEditions, 2003

${ }^{9}$ Cf. Laurence Kaufmann, «Entre fiction et réalité. L'opinion publique dans la France du XVIIIème siècle », in Javier Fernandez Sebastian et Joëlle Chassin coord., L'avènement de l'opinion publique. Europe et Amérique XVIIIème - XIXème siècle, Paris, L'Harmattan, 2004

${ }^{10}$ Voir sur ce point notre récent ouvrage, Discours et événement. L'histoire langagière des concepts, Presses Universitaires de France Comté, 2006, en particulier la Postface.

${ }^{11}$ Voir sur ce point André Charrak, Empirisme et métaphysique. L « Essai sur l'origine des connaissances humaines » de Condillac, Paris, Vrin, 2003
} 
Désormais inscrite dans la logique de la nature humaine, y compris par le fonctionnement du cerveau, une philosophie de l'esprit se traduit ici dans une véritable psychologie cognitive ${ }^{12}$. Cependant une telle dynamique de l'esprit, et les divers processus discursifs qui en découlent, confèrent à l'appareil mental, au-delà de son internalité, un caractère à la fois unitaire et fragmenté. Unitaire par la mise en valeur d'un « sujet exprimant » au titre du rôle central du langage. Fragmenté verticalement par le fait de la distinction entre un substratum moi (inconscient pour une grande part, ou tout du moins incogniscible, donc distinct de la perception consciente - l'aperception - associée à un jugement), et des manières d'être, d'agir et de penser, qui laissent de côté une multitude de petites perceptions imperceptibles pour la conscience.

A l'école de la Révolution française, cet appareil mental prend un tour nettement culturel, voire institutionnel dans le sens où les élites révolutionnaires s'efforcent d'imprimer à l'imagination une direction rationnelle $\mathrm{e}^{13}$. Il s'agit de promouvoir une politique républicaine qui inscrit l'imagination individuelle dans une dynamique sociale en l'associant étroitement au sentiment patriotique, à l'exemple des fêtes civiques. Une nouvelle fois, Condillac, plus que Rousseau, occupe une place centrale dans une telle part analytique de la Révolution française qui cultive les facultés mentales à des fins sociales, et principalement l'imagination, et de manière singulièrement intentionnelle. Mais il s'agit là plus du résultat d'un processus que d'un état de fait imposé par les événements politiques. La question demeure de savoir à quel moment l'internalité de l'appareil mental, connecté à l'externalité du référent sociologique, se traduit dans un travail de l'esprit politique qui permet de rendre pensable et possible le fait révolutionnaire

Faut-il alors considérer qu'il n'existe pas vraiment un moi perçu en tant que tel dans l'appareil mental, le moi n'existant que de façon fragmentée pendant la Révolution française et ne prenant donc un tour véritable unitaire qu'avec l'ordre bourgeois, et sa philosophie au travers de l'œuvre de Victor Cousin ${ }^{14}$ ?. Nous ne le pensons pas dans la mesure où, si « l'école de Condillac », expression désignant les Idéologues, tend bien à postuler une passivité et une fragmentation du moi, c'est pour contrecarrer la métaphysique du moi et de son activité des révolutionnaires

Ainsi le moment nominaliste des années 1770-1780 introduit bien une figure de l'individu social, puis de l'individu-nation en 1789, sous couvert de l'individu empirique, où l'on trouve formulé, plus particulièrement sous la plume de Sieyès, une métaphysique du moi et de son activité. Dès le Grand cahier métaphysique (1773), Sieyès propose bien une approche cognitive de nature génétique et d'inspiration condillacienne, en matière de formation du moi. Il met en œuvre un schéma cognitif qui permet « la reconnaissance du moi », formulation certes plus tardive dans la mesure où se schéma se perpétue jusque dans ses écrits des années 1810 en particulier les Vues analytiques), ne serait-ce que dans une autre reformulation, celle du « principe d'activité » dans « la force vitale ${ }^{15}$. Le moi se présente dans sa dimension verticale, c'est-à-dire au titre de la différence entre le " moi absolu », " moi substratum », " moi véritable » situé hors des phénomènes donc inaccessible à la connaissance humaine, et « le moi de chacun » qui se décline selon divers points de vue, génétiquement liés : le moi

12 Voir sur ce point Jane Goldstein, The Post-Revolutionary Self. Politics and Psyche in France (1750-1850), Harvard University Press, 2005.

13 Jan Goldstein consacre à cette question un chapitre de son livre, Ibid.

${ }^{14}$ Jan Goldstein aborde également ce point avec précision, Ibid.

${ }_{15}$ Voir notre édition du Grand cahier métaphysique et des Vues analytiques dans Des Manuscrits de Sieyès, volume 1 et 2, sous la dir. de Christine Fauré, Paris, Champion, 19992007, ainsi que notre ouvrage Sieyès et l'ordre de la langue, Paris, Kimé, 2002. 
sentant, le moi percevant, le moi exprimant, le moi voulant, le moi apercevant et le moi jugeant.

Si la nouvelle métaphysique du moi procède de la généalogie de la marche de l'esprit humain, donc de l'ordre et de l'arrangement des opérations de l'esprit, c'est dans la mesure où elle se veut restreinte à l'expérience humaine, permettant ainsi de requalifier la connaissance de nos manières d'être, de penser et d'agir se situant au plus près des données empiriques et historiques. A ce titre, elle dispose, en particulier au cours du moment nominaliste des années 1770 et 1780 , d'un socle sociologique associant vérité du moi et utilité sociale, avant même de s'inscrire dans l'horizon, propice à l'action, du droit naturel déclaré, puis réalisé

Cette métaphysique ne sera pas retenue par la philosophie « officielle » du Directoire sous l'emprise des Idéologues, tout en demeurant, nous semble-t-il, la clé de la compréhension philosophique de l'événement national constitutif de la Révolution française.

\section{II - L’idéologie proprement dite}

La période directoriale, un moment idéologique.

La période de la République directoriale, durant laquelle s'invente la notion d'idéologie, marque un renversement important de la conception du politique par rapport à la période antérieure centrée autour du déploiement d'une métaphysique politique dans l'espace du pouvoir législatif sur la base d'un socle sociologique pensé dès les années 1780. La politique pratique prend le dessus sur la théorie pratique du droit naturel ${ }^{16}$, au nom de l'établissement d'un pouvoir exécutif stable, et permet ainsi la partition entre une idéologie politique sans contenu novateur et l'idéologie comme science expérimentale ${ }^{17}$.

En effet, durant le Directoire, les hommes politiques au pouvoir s'efforcent de garantir le pouvoir exécutif par la multiplication de pratiques empiriques guidées par une idéologie du juste milieu. Ainsi s'impose progressivement une idéologie politique peu soucieuse de renouvellement doctrinal, donc un pragmatisme idéologique de la part d'un nombre grandissant d'acteurs politiques qui, véritables girouettes, vident le sens du jeu politique en le réduisant à un marchandage d'idées et à la conquête de biens matériels. La politique " globale » devient alors une affaire de gestion objective de la contingence à l'écart du travail antérieur de l'esprit politique. Révoquant désormais tout artificialisme, elle se donne alors dans la profusion des textes et des prises de parole de manière à créer un maximum de visibilité à l'aide d'un minimum de conceptualisation des programmes politiques.

Ainsi se met en place, au centre de l'échiquier politique, une position raisonnable sans véritables dépositaires idéologiques - un vide à remplir donc par un déplacement vers la pensée savante -, mais très en prise sur une idéologie de l'ordre public. De fait, le temps de la conquête de l'espace parlementaire, et son corollaire la promotion de la métaphysique politique au fondement du pouvoir législatif, sont révolus au nom d'un intérêt de plus en plus exclusif pour le pouvoir exécutif. Bien sûr, il existe un courant démocratique pendant le Directoire, dont Bernard Gainot s'est fait $1^{\prime}$ historien ${ }^{18}$ : les néo-jacobins d'en prennent au pragmatisme des « hommes d'Etat», en dénonçant les apories de leur appui sur un principe conservateur de l'ordre social. Mais ils ne connaissent que des demies victoires, participant

\footnotetext{
${ }^{16}$ Voir à ce propos Florence Gauthier, Triomphe et mort du droit naturel en Révolution, 17891795-1802, Paris, PUF, 1992.

${ }^{17}$ Voir en particulier, à ce sujet, l'ouvrage de Pierre Serna, La République des girouettes, Paris, Champvallon, 2005.

${ }^{18}$ En particulier dans 1799, un nouveau jacobinisme? Paris, Editions du CTHS, 2001.
} 
ainsi, sans doute à leur corps défendant, d'un même climat idéologique fait d'instrumentalisation de l'opinion et de théâtralisation des gestes démocratiques.

Dans un tel contexte, les savants du Directoire trouvent alors leur assise sociale dans la promotion de mécanismes idéologiques liés aux circonstances avec pour objectif la formation et le renforcement de l'appareil d'Etat, ce qui leur laisse une grande marge de manœuvre dans la formulation de leur discours scientifique propre, plus particulièrement en terme d'idéologie. A vrai dire, Jean-Luc Chappey a bien montré qu'une telle conception du travail savant, formulé à l'aide de l'argument "science de l'homme», est pensée avant la Révolution française, et en ses débuts, mais demeure en retrait par rapport à l'investigation plus politique en terme d'art social. C'est donc à partir de 1795, avec la République directoriale, que se met en place, toujours au titre de « la science de l'homme », " un véritable paradigme, un horizon d'intelligibilité qui devient envahissant autant dans l'espace des savoirs que dans le monde politique, s'incarnant au sein de la nébuleuse bien connue des Idéologues ${ }^{19}$.

Nouvelle science qui se veut donc «science du gouvernement» au plus près du pouvoir exécutif par contraste avec «la science politique » du législateur philosophe, elle s'inscrit dans le contexte du rétablissement de l'ordre public tant par la répression que par un souci de normalisation des principes à la base du savoir. Ainsi le régime directorial fonde son projet politique sur la construction d'une science, l'idéologie, permettant de le légitimer par la normalisation de l'œuvre de régénération sociale

Appartenant au centre républicain, délestés de l'héritage métaphysique si proche, associés à la modération politique dominante, les savants peuvent révoquer «l'art social » et son «socle sociologique », cette combinaison particulière d'artificialisme et d'empirisme, et associer l'art $d u$ compromis, véritable idéologie pratique, à une science expérimentale définie dans le terme même d'idéologie. En un sens, le Directoire invente le terme d'idéologie à la fois dans la chose politique et dans le mot scientifique. Les Idéologues inventent l'idéologie dans les mots de la science par analogie avec le contexte d'un projet politique foncièrement idéologique.

\section{L'invention de l'idéologie (Destutt de Tracy)}

Dès 1797, dans un article du Moniteur Universel intitulé Sur un système méthodique de bibliographie, Destutt de Tracy précise ce qu'il entend par idéologie, « l'analyse de la pensée tout simplement ${ }^{20}$. C'est donc de façon discrète, sous couvert de l'analytique condillacienne, que le terme est introduit. Cependant, Destutt aborde plus avant les avantages de cette nouvelle dénomination, et en donne une définition précise dans son Mémoire sur la faculté de penser de 1798, tout en précisant les circonstances de son énonciation :

«La science qui nous occupe est si neuve, qu'elle n'a pas encore de nom (...) Toute science est le produit de l'analyse d'un sujet, et non pas cette analyse elle-même. Le produit de l'analyse des sensations et des idées n'est donc pas nommé. Il ne peut être appelé métaphysique. Ce mot désigne une science qui traite de la nature des êtres, des esprits, des différents ordres d'intelligence, de l'origine des choses, de leur cause première (...) Il est vrai que l'on peut dire que, ne connaissant rien que par nos sensations, nos sensations étant tout pour nous, nous pouvons appeler physique la connaissance de nos sensations considérées dans tous les êtres qui les occasionnent; et métaphysique, la connaissance de ces mêmes sensations considérées dans leurs effets en

${ }^{19}$ «De la science de l'homme aux sciences humaines : enjeux politiques d'une configuration de savoir (1770-1808) », Revue d'histoire des sciences humaines, Naissance de la science sociale (1750-1850), $\mathrm{N}^{\circ} 15,2006$, p. 54.

${ }^{20}$ Mémoire sur la faculté de penser et autres textes, Corpus, Fayard, 1992, p. 27. 
nous. Sous ce point de vue, l'une est l'histoire du monde, et l'autre est l'histoire de notre $m o i$, du petit monde. Cette division est belle et complète. Mais c'est là un emploi tout à fait nouveau du mot métaphysique; et ce mot est si cruellement discrédité que je verrai avec peine qu'on s'en servit pour désigner la science de la pensée (...) Je préférerais donc de beaucoup que l'on adoptât le nom d'idéologie, ou science des idées (...) Car idéologie est la traduction littérale de science des idées » (p. 70-71) ${ }^{21}$.

Utiliser le terme d'idéologie, c'est donc « éviter d'employer le terme de métaphysique », tel est le point central que Destutt développe d'emblée. Il s'agit non seulement de rompre avec la métaphysique théologique, mais aussi de penser l'idéologie contre la métaphysique du moi et de son activité, telle qu'elle s'est mise en place par requalification ontologique des connaissances au fur et à mesure de la classification encyclopédiste des sciences nouvelles, et telle qu'elle s'est concrétisée, dès les années 1770-1780, dans une science politique.

Destutt, considérant que « la science de la pensée n'a pas encore de nom », lui donne alors celui d'idéologie. Ainsi « la science des idées » est « science de l'idéologie » dans la mesure où « le nom d'idéologie » désigne à la fois le but, la connaissance de l'homme, et les moyens, l'analyse de ses facultés, de cette science. Destutt précise également, dans son Mémoire de 1798 , que ce n'est pas tant la part " psychologique » de l'idéologie, encore très mystérieuse et confrontée à de nombreuses erreurs, que la part rationnelle de l'idéologie qui l'intéresse dans la mesure où elle se borne d'elle-même. Ainsi il ajoute: "Je prends nos facultés telles qu'elles sont et m'occupent que de leurs effets ». Il considère ainsi que l'idéologie est essentiellement «une science expérimentale»: elle permet de dresser le constat de l'importance des "phénomènes idéologiques », et de considérer plus les applications de l'idéologie que sa théorie si tant est qu'elle existe. "Points fondamentaux 》 et "vérités nouvelles» en idéologie ponctuent alors ses Eléments d'idéologie en plusieurs volets, Idéologie proprement dite, Grammaire, Logique, enfin le Traité de la volonté et de ses effets, entre 1801 et 1815. Destutt en vient ainsi à définir l'idéologie comme «la science de la formation de nos idées », des « opérations de notre intelligence ».

Reste à faire un constat très important : les idéologues positionnent en second le langage par rapport aux idées, ce qui revient à récuser le fait que la langue (politique) peut être une institution sans analogue, donc légitimée par les seuls acteurs. Ils affirment alors que les signes ne peuvent fonder le progrès des idées ce qui revient à définir l'idéologie comme " langue bien faite » au sens condillacien, tout en la dissociant de la langue politique en tant que traduction de la métaphysique philosophique. Or le fondement ontologique de la réalité dans les signes permet de laisser ouvert ce qui doit et peut être dit dans l'ordre des possibles. Ainsi la validité de l'observation sociale ne se déploie plus dans un travail de l'esprit qui propose une nouvelle systématicité conceptuelle sur la base de ce qui doit être avec une part majeure accordée à la créativité linguistique. Le travail du savant se réduit désormais à analyser une systématicité déjà donnée de faits : seul est de son ressort le tableau « exact » des faits. La « révolution de l'analyse » des Idéologues, tâche à la fois politique et scientifique, est une révolution à sens unique, dans la mesure où elle pose les bases du nouveau système social en le décrochant de la métaphysique politique de la Révolution française. La voie est ouverte pour " une science de l'homme » prenant nom d' "anthropologie», chez Cabanis, dans l'ordre d'une société qui est l'ordre dans la société où cette science est établie ${ }^{22}$.

\footnotetext{
${ }^{21}$ Ibid., pp. $70-71$.

22 Voir sur ce point le recueil de textes commentés de Laurent Clauzade, L'idéologie ou la révolution de l'analyse, Paris, Tel-Gallimard, 1998, et l'étude de Daniel Teysseire, "Lien social et ordre politique chez Cabanis », Studies on Voltaire and the Eighteenth Century, 1989,41, p. $353-400$.
} 
«L'école de Condillac » renvoie alors l'usage de signes artificiels tout la fois archétypique et imperceptible dans nos jugements à la part d' "obscurité de l'idéologie », par l'effet de l'habitude. C'est bien là où se révèle les limites d'une telle entreprise politique. Si l'idéologie peut s'avérer être « un corps de doctrine assuré et déjà fort étendu » ${ }^{23}$, voire " une moderne métaphysique philosophique» en complète opposition avec " la métaphysique théologique ${ }^{24}$; il n'est pas si simple de rompre avec les connaissances antécédentes à la découverte de l'idéologie, surtout lorsqu'elles sont issues d'une requalification récente de la métaphysique auprès d'une théorie expérimentale de la connaissance. Méditons alors, convient Destutt, sur l'attitude suivante de pensée : « revenons à l'observation de nous-même, à l'idéologie », au risque de sortir l'étude de l'esprit humain du carcan de l'idéologie !

Sous couvert d'invention - certes le terme d'idéologie est bien ici un néologisme -, nous nous trouvons donc devant une tentative particulièrement systématique et conservatrice de faire obstacle à la métaphysique politique de la Révolution française, déployée de Sieyès à Robespierre tant dans son fondement avec la métaphysique du moi que dans sa quête d'une langue philosophique en adéquation avec le mouvement national et/ou populaire. Une rupture à vrai dire sans lendemain dans la mesure où les penseurs libéraux qui succèdent aux Idéologues, en particulier les Doctrinaires, renouent le fil avec la métaphysique du moi contre les Idéologues, certes à leur façon proprement idéaliste. Maine de Biran occupe alors une place centrale dans la manière libérale de poser les limites de l'idéologie, peu de temps après sa formulation.

Les limites de l'idéologie (Maine de Biran).

Au départ, Maine de Biran profite de l'invention de l'idéologie par Destutt de Tracy pour «transporter la physiologie dans l'idéologie », c'est-à-dire s'efforce d'unir l'idéologie à la physiologie dans les termes suivants :

«L'idéologie peut s'élever au-dessus de la grammaire générale et pénétrer des formes extérieures de la pensée ou des signes dont elle se revêt, jusqu'à ces modifications plus intimes, plus profondes dont les signes se tirent uniquement de la connaissance des lois de notre organisation $»^{25}$.

Mais, à s'intéresser aux modifications profondes de l'individualité, Maine de Biran se détache de plus en plus des " philosophes sensualistes » à qui il reproche de réduire l'individu à ses besoins et ses droits. Il en vient tout particulièrement dans son Journal des années 1814$1817^{26}$ à réhabiliter le travail métaphysique contre les Idéologues sur la base d'une réflexion philosophique sur le moi. L'opportunité lui en est ouverte par les discussions au sein d'une " société philosophique » où il est question de « notre doctrine commune sur le sentiment du moi, de l'activité, etc. ${ }^{27}$ avec Royer-Collard, Guizot, Degérando et d'autres. En centrant son attention sur «le moi et le fait primitif de la conscience», Maine de Biran en vient à considérer, sur la base d'un principe de vie situé en amont de l'activité de l'individu, " une suite de phénomènes sensitifs » situés hors des phénomènes idéologiques. Il s'agit alors de proposer une « doctrine psychologique » centrée sur « les impressions affectives dénuées de

${ }^{23}$ Dissertation sur quelques questions d'idéologie, in Mémoire sur la faculté de penser et autres textes, op.cit., p. 200

${ }^{24}$ Ibid., Dissertation sur l'Existence, p. 206.

${ }^{25}$ De l'influence de l'habitude sur la faculté de penser, 1802, p. 195

${ }^{26}$ Journal, 3 volumes, Neuchâtel, Editions de la Baconnière, 1954-1957.

${ }^{27}$ Ibid., I, 19 
personnalité $»^{28}$, et distincte d'une psychologie expérimentale liée à l'Idéologie qui s'en tenait « à la perception claire, à l'époque de la distinction du moi et de ses modifications » ${ }^{29}$.

Comme l'indique fort justement Anne Devarieux ${ }^{30}$, Maine de Biran propose une psychologie en tant que métaphysique de l'expérience intérieure, une philosophie qui identifie, dans l'expérience intérieure, le moi et l'effort dans une reprise en charge permanente de soi. Ainsi il conceptualise un espace subjectif par la mise en évidence d'une sorte d'étendue intérieure. Il rend compte de l'immédiateté de la sensibilité personnelle dans l'unité sentie et donnée d'un fait primitif à l'origine de la sensibilité affective et intuitive.

De ce fait, il considère le temps de l'idéologie comme obsolète dans les termes suivants :

« Lorsque l'on a essayé de nos jours d'introduire le mot nouveau idéologie à la place du terme de métaphysique qu'on proscrivait, on a pu annoncer qu'on ne voulait plus s'occuper de ces hautes spéculations de la métaphysique proprement dite (...) Il était utile d'avoir un terme quelconque qui fixât la ligne de démarcation. Mais quand on a prétendu remplacer entièrement la métaphysique par l'idéologie, on a cru pouvoir soumettre au point de vue physique ou logique ces conceptions ou notions premières et nécessaires que la métaphysique a essentiellement pour objet; on a tout confondu, on est tombé dans des erreurs graves » (p. 43) ${ }^{31}$.

Un temps utile pour se dissocier de la métaphysique politique de la Révolution française, le terme d'idéologie perd de sa pertinence du moment où le retour au travail métaphysique, sur la base de l'observation intérieur du moi, permet l'émergence du « moi bourgeois ». C'est à Victor Cousin que nous devons alors l'expansion d'une conception subjective de la volonté individuelle inscrite dans la lignée des réflexions de Maine de Biran, mais articulée avec l'objectivité de la raison impersonnelle. Ainsi le «moi général » est « un moi identique et par conséquent un, sous la variété de ses actes, de ses pensées, de ses sensations ${ }^{32}$. S'il convient donc de retenir, dans l'histoire de la philosophie, « l'expression récente et célèbre d'idéologie pour désigner la science de l'entendement humain »; il n'est reste pas moins, précise Victor Cousin, qu' «il s'agit maintenant de passer aux applications de l'idéologie, à la connaissance des objets et des êtres à l'aide des idées ${ }^{33}$.

\section{Conclusion: Napoléon et les Idéologues}

En mettant l'accent sur le fait que l'idéologie a voulu s'imposer contre la métaphysique politique, nous avons quelque peu déplacé l'opinion courante selon laquelle le terme d'idéologie perd pied sur le terrain scientifique sous les coups de boutoir de Bonaparte qui, en le prenant dans un sens péjoratif, le transforme en injure, et le donne ainsi en héritage au XIXème siècle.

Qu'en est-il ?. Dans le Journal de Paris du 15 Pluviôse an IX, il est écrit que Bonaparte s'en serait pris aux Idéologues en tant que partisans d'une « métaphysique politique » dissociée de la vérité des droits de l'homme et de la liberté de conscience, Puis, un an plus tard, Bonaparte attaque de nouveau cette " classe de phraseurs et d'idéologues », condamnant ainsi l'idéologie dans les termes suivants: "C'est à l'idéologie, à cette ténébreuse métaphysique qui, en

${ }^{28}$ Ibid., I, 27

${ }^{29}$ Ibid., I, 79.

${ }^{30}$ Maine de Biran. L 'individualité persévérante, Paris, Jérôme Millon, 2004.

${ }^{31}$ Journal, op. cit. , II, 43.

${ }^{32}$ Cours de l'histoire de la philosophie. Histoire de la philosophie morale au $18^{\text {ème }}$ siècle, $1829,2,432$.

${ }^{33}$ Ibid., 2, pages 104 et 296. 
recherchant avec subtilité les causes premières, veut sur ces bases fonder la législation des peuples, au lieu d'approprier les lois à la connaissance du cœur humain et aux leçons de l'histoire, qu'il faut attribuer tous les malheurs qu'a éprouvés notre belle France » ${ }^{34}$.

Pierre Macherey, dans une étude sur « La péjoration de l'idéologie, de Napoléon au Jeune Marx $»^{35}$, en déduit que le terme prend ainsi un tour péjoratif, en nous renvoyant au phraseur, au manipulateur d'idées, au fauteur d'illusion, et marque fortement Marx, très vite obnubilé par de tels aspects négatifs de l'idéologie, retardant ainsi sa prise de conscience de la nécessité d'une pensée, pour ne par dire d'une théorie de l'idéologie en tant que telle. L'étude plus approfondie de Hyondok $\mathrm{Choe}^{36}$ arrive à la même conclusion sur le sens majoritairement péjoré du mot idéologie.

Il est vrai aussi que les écrivains français des années 1810-1830 tendent à véhiculer une même interprétation de la forte imprégnation du terme d'idéologie par le contexte "napoléonien ». Deux exemples. Dans son Histoire parlementaire de la Révolution française, Buchez note la phrase suivant de Garat à la séance de la Chambre du 5 juillet 1815 : «Avez-vous entendu ce mot d'idéologie? Il rappelle un fait aussi, et très important : c'est qu'au moment où l'on voulut nous imposer le pouvoir absolu, ce même mot fut frappé de toute la défaveur du trône ». En 1818, Madame de Staël remarque que Bonaparte " avait pris en horreur ce mot très innocent, parce qu'il signifiait la théorie de la pensée ${ }^{37}$. Stendhal, dans Lucien Leuwen (1834), constate « les injures que Napoléon adressa à l'idéologie de M. Tracy ».

Le dossier semble donc clos par amassement de preuves: Bonaparte serait profondément hostile à la métaphysique politique de la Révolution française ; il voit dans les Idéologues, à tort bien sûr, les représentants de ce courant métaphysique, il en vient ainsi à donner, pour de nombreuses années, le ton majoritaire sur la péjoration de l'idéologie. Notre étude voudrait au moins inciter les chercheurs à réinterroger la question.

Il faut d'abord savoir que Bonaparte ne dédaignait pas les discussions métaphysiques, en particulier avec Sieyès qui défend l'existence nécessaire d'une métaphysique politique contre les Idéologues ${ }^{38}$. Par ailleurs, en proclamant « Je suis national », Napoléon considère qu'il a « fini » le tout de la nation ${ }^{39}$ conceptualisé par Sieyès dès 1789 au nom de la métaphysique politique, comme en témoigne un échange laconique avec Sieyès retranscrit dans le Mémorial de Sainte-Hélène $e^{40}$. Ainsi, son hostilité à la métaphysique des Idéologues nous renvoie peutêtre, par le seul fait de la qualifier péjorativement d'idéologie, à une critique d'une version

${ }^{34}$ Voir sur ce point Georges Gusdorf, La conscience révolutionnaire. Les Idéologues, Paris, Payot, 1978, en particulier pages 320-321.

${ }^{35}$ Disponible sur le Web dans les archives du groupe d'étude « La philosophie au sens large » de l'UMR Savoirs/Textes/Langage. Voir également Emmet Kennedy, "'Idéologie' from Destutt de Tracy to Marx », Journal of the History of Ideas, Vol. 40, №3 (1979), p. 353-368.

36 Ideologie. Eine Geschichte der Entstehung des gesellschaftskritischen Begriffs, Frankfurt/M, Peter Lang, 1997

${ }^{37}$ Dans les Considérations sur les principaux événements de la Révolution française, édition Delaunay, p. 706.

${ }^{38}$ Le biographe de Sieyès, Jean-Denis Bredin, relate diverses occasions où Bonaparte et Sieyès parlent métaphysique, Sieyès. La clé de la Révolution française, Paris, Editions du Fallois, 1998 , p. 401

${ }^{39}$ Voir sur ce point, Didier Le Gall, Napoléon et le Mémorial de Saint-Hélène, Paris, Kimé, 2003, en particulier le chapitre II

${ }^{40}$ « Eh bien, vous voici pêle-mêle avec les anciens ducs et les anciens maquis, regardez-vous le tout comme fini ? Oh! oui, dit Sieyès, s'inclinant profondément, vous avez accompli des prodiges que rien n'égale, et qu'il était au-dessus de mes forces de prévoir », Las Cases, Mémorial de Sainte-Hélène, Editions du Seuil, 1968, p. 516. 
édulcorée, en particulier des droits de l'homme, de l'idéologie sous couvert d'hostilité à la métaphysique.

En appliquant aux Idéologues un terme qu'ils récusent, celui de métaphysique, du moins dans sa composante politique, Napoléon leur retourne leur stratégie d'effacement de la métaphysique politique en les accusant eux-mêmes d'en être les phraseurs, c'est-à-dire d'en vider le contenu de connaissance tel qu'il s'est inscrit à l'horizon des droits de l'homme et du citoyen. Le non-dit de l'idéologie, dans le temps de son invention, c'est donc aussi une certaine absence du langage de la vérité - référence partagée par Sieyès et Napoléon - dans le fait de mettre au premier plan la duperie de soi pour mieux duper les autres ${ }^{41}$. Les Idéologues se baignent dans l'espoir de tromper Napoléon en promouvant la science comme idéologie alors qu'ils ont le but bien plus pragmatique, plus idéologique d'imposer «les principes abstraits de la liberté civile 》 - termes «napoléoniens »-, donc leur conception d'un Etat républicain à l'encontre du « tout de la nation » que Napoléon veut incarner dans sa personne. Napoléon n'est pas dupe, et leur retourne à sa façon l'argument en les accusant d'être des métaphysiciens qui s'ignorent, et pire encore d'être, à l'instar des jacobins, des partisans de l'insurrection et de la souveraineté du peuple. Dans cet univers de démentis silencieux, si l'on peut dire, la partie d'idéologie n'est pas facile. Une affaire à suivre de plus près, donc.

${ }^{41}$ Voir à ce propos l'ouvrage collectif Figures de la duperie de soi, sous la dir. De Augustin Giovannoni, Paris, Kimé, 2001. 